\title{
Obligatorische Blockkurse 2002 für die Erlangung des Facharzttitels FMH Gynäkologie und Geburtshilfe Cours-bloc obligatoires 2002 pour l'obtention du titre de spécialiste FMH en gynécologie-obstétrique
}

\author{
Kurs Nr. 1: Benigne und prämaligne Erkrankung \\ des unteren Genitalbereiches \\ Datum: Samstag, 6. April 2002 \\ Ort: Kantonsspital Bruderholz \\ Leitung: Prof. Dr. med. S. Heinzl \\ Auskunftsstelle: Chefarztsekretariat Frauenklinik, \\ Tel. 06143621 83, Fax 06143636 66, E-Mail: \\ elke.leuenberger@ksbh.ch
}

\author{
Cours No 2: Gynécologie et médecine \\ de la fillette et de l'adolescente \\ Date: samedi 25 mai 2002 \\ Lieu: Lausanne \\ Direction: Prof. Dr P. De Grandi, \\ Prof. Dr P.-A. Michaud, Dr S. Renteria \\ Information: Secrétariat Unité multidisciplinaire de \\ la santé des adolescents UMSA, tél. 02131437 60, \\ fax 02131437 67, e-mail: umsa@chuv.hospvd.ch
}

\author{
Cours No 3: Colposcopie: \\ diagnostic et traitement en 2002 \\ Date: samedi 5 octobre 2002 \\ Lieu: Genève \\ Direction: PD Dr A. Major \\ Information: Secrétariat Dr Major, Mme Georgette \\ Moret, tél. 02238243 22, fax 02238240 30, \\ e-mail: georgette.moret@hcge.ch
}

\author{
Kurs Nr. 4: Pränatale Diagnostik \\ Datum: Samstag, 23. November 2002 \\ Ort: Zürich \\ Leitung: PD Dr. med. R. Zimmermann \\ Auskunftsstelle: PD Dr. med. Roland Zimmermann, \\ Tel. 0125550 88, Fax 012554430
}

Die Kurse 1 und 4 wenden sich schwergewichtig an Assistenten/-innen, welche am Anfang der Weiterbildung stehen, die Kurse 2 und 3 an Fortgeschrittene ab 3. Jahr spezifischer Weiterbildung.

Les cours 1 et 4 sont destinés aux assistant(e)s en début de formation, les cours 2 et 3 aux assistant(e)s à partir de la $3^{\mathrm{e}}$ année de formation spécifique. 